\title{
Face Recognition Feature Comparison Based SVD and FFT $^{*}$
}

\author{
Lina Zhao, Wanbao Hu, Lihong Cui
}

School of Science, Beijing University of Chemical Technology, Beijing, China.

Email: zhaoln@mail.buct.edu.cn, \{huwanbao2008,mathcui2\}@163.com

Received January $5^{\text {th }}, 2012$; revised February $28^{\text {th }}, 2012$; accepted March $31^{\text {st }}, 2012$

\begin{abstract}
SVD and FFT are both the efficient tools for image analysis and face recognition. In this paper, we first study the role of SVD and FFT in both filed. Then the decomposition information from SVD and FFT are compared. Next, a new viewpoint that the singular value matrix contains the illumination information of the image is proposed and testified by the experiments based on the ORL face database finally.
\end{abstract}

Keywords: Face Recognition; Feature Extraction; SVD; FFT

\section{Introduction}

As the most natural and important characteristic, face plays the central role in various forms of human biological characteristics. Since 1970s, Face recognition has been attracting overwhelming interests in the field of image engineering and computer vision. Until now, face recognition technology has gone through three stages of change: The first stage (1964-1990) is usually only as a general pattern recognition problem to study; In the second stage (1991-1997), face recognition technology gained rapid development, the proposed algorithm greatly improved the efficiency and accuracy; In the third stage (1998-now), face recognition technology gradually tend to mature, research focuses on solving the problems of poor robustness. The mainstream technology of face recognition basically is divided into three categories [1], the approach based on geometry feature [2], based on template matching method [3-5] and based model [6,7].

The general steps for face recognition are as follows. The first step is image acquisition and the source of face information can get form scanning a photo by scanner, shooting face by a digital camera and face database. The second step is face detection and location which means processing and analysis input image to determining whether there is one face. If there are people faces, we should find the location of the face in the image, and separate face from the background image. The third step is image

\footnotetext{
"This work was partially financed by the following projects: Tianyuan Fund of National Natural Science Foundation of China (10926187), Central University special fund basic scientific research and operating expenses (ZZ1019), National Natural Science Foundation of China (60803099).
}

pre-processing and the main role of image preprocessing is to remove or reduce the interference so that provide high-quality image. The interference may be caused from the light, the imaging system and the external environment. The most commonly used methods contain geometric normalization and elimination of noise. The fourth step is feature extraction and selection and this module's role is to extract feature from the image that is pre-processed, then the original image data is mapped to feature space. Since the original image data is quite large, it is necessary to transform the original data to effectively achieve the classification and recognition. The fifth step is training and this process may also be known as the classifier design. The end of this process will generate the parameters can be used to identify. In this part, the basic approach is to determine a decision rule based on the sample training set, so that when we classify objects, error recognition rate or error is the smallest. The last step is identification. According to the parameters derived from training, the module is used to complete the work of human face discrimination, give the final recognition results.

Although face recognition has been developed so many years and there are so many mature algorithms and technologies, but there are still difficulties in the procedures. Recognition of face images acquired in an outdoor environment with changes in illumination and or pose remains a largely unsolved problem. In other words, current systems are still far away from the capability of the human perception system [8]. How about decomposing image into two parts: illumination information and texture and pose information?

In this paper, we do the following work: we elaborate 
the Fourier transform and SVD of the image in Section 2. We compare the action between two decompositions. FFT transform image to amplitude spectrum and phase spectrum, SVD decompose the image matrix into singular matrix and singular vector matrixes. We propose that singular matrix contains the same illustration information just like amplitude spectrum does. We design three experiments and testified our proposal in Section 3. Section 4 is the conclusion of our work.

\section{The Decomposition of the Image}

\subsection{Fourier Transform of Image}

Some functions which meet certain conditions can be expressed as a trigonometric function or their integral linear combination by Fourier transform. In different research areas, Fourier transform has many different variations, Such as the continuous Fourier transform and discrete Fourier transform.

Here we give the definition:

If $f(t)$ has the following form

$$
f(t)=\frac{1}{2 \pi} \int_{-\infty}^{+\infty}\left[\int_{-\infty}^{+\infty} f(\tau) e^{-i \omega \tau} \mathrm{d} \tau\right] e^{i \omega t} \mathrm{~d} \omega
$$

Then

$$
\begin{gathered}
F(\omega)=\int_{-\infty}^{+\infty} f(t) e^{-i \omega t} \mathrm{~d} t \\
F^{-1}[F(\omega)]=\frac{1}{2 \pi} \int_{-\infty}^{+\infty} F(\omega) e^{i \omega t} \mathrm{~d} \omega
\end{gathered}
$$

$F(\omega)$ is called the Fourier transform of $f(t)$; $F^{-1}[F(\omega)]$ is called inverse Fourier transform of $F(\omega)$.

\subsection{Spectrum Analysis}

After the discrete Fourier transform, we can obtain amplitude spectrum and phase spectrum of the image. In the image detection, image feature detection and texture analysis is an important direction. Amplitude spectrum represents relation between the signal amplitude and frequency, phase spectrum represents relation between initial phase of the signal and frequency.

Two-dimensional Fourier transform:

$$
\begin{aligned}
& F(u, v) \\
& =\frac{1}{M N} \sum_{m=0}^{M-1} \sum_{n=0}^{M-1} f(m, n) \exp \left[-i 2 \pi\left(\frac{m u}{M}+\frac{n v}{N}\right)\right], \\
& u \in(0, M-1), v \in(0, N-1)
\end{aligned}
$$

Two-dimensional inverse Fourier transform:

$$
\begin{aligned}
& f(m, n) \\
& =\sum_{m=0}^{M-1} \sum_{n=0}^{M-1} F(u, v) \exp \left[-i 2 \pi\left(\frac{u x}{M}+\frac{v y}{N}\right)\right], \\
& x \in(0, M-1), y \in(0, N-1)
\end{aligned}
$$

After a Fourier decomposition of the image, the phase spectrum contains texture and Structure information about the image, the amplitude spectrum saves contrast illumination information [9].

There are many main properties of the Fourier transform, for example, symmetry, linear, odd-even and actual situation, scale transformation, time-shift feature, frequency shift, differential properties, time-domain integral. Because of the good nature, Fourier transform have a wide range of applications in many fields, such as physics, number theory, signal processing, probability, statistics, cryptography, acoustics, optics, etc.

Fast Fourier transform is the fast algorithms of the discrete Fourier transform. FFT lies at the heart of signal and image processing fields, encompassing a versatile range of applications, such as telecommunications, medical imaging, and spectral analysis.

\subsection{Singular Value Decomposition of the Image}

\subsubsection{The Significance of Singular Value Decomposition Algorithm}

Effectiveness of all methods of face recognition depends on two aspects: feature representation and feature matching [10], feature representation is the pattern feature extraction in pattern recognition. The priority of image recognition is extract effective image feature. In [2], image features had been divided into four categories: visual features, the demographic characteristics, characteristics of transform coefficients, algebraic features. The algebraic features of the image reflect an intrinsic property of the image; it is stable in a certain range. Hong [11] presented identification method based on singular value decomposition (SVD). The method provides a new algebraic method for extracting image algebraic features. Effectiveness of this method has been verified in [11-13].

\subsubsection{Singular Value Decomposition Theorem}

If $\boldsymbol{A} \in R_{r}^{m \times n}(r>0)$, there exists orthogonal matrix $\boldsymbol{U}$ of $m$ order and orthogonal matrix $\boldsymbol{V}$ of $n$ order,

$\sigma_{i}(i=1,2, \cdots, r)$ is non-zero singular value of the matrix $\boldsymbol{A}$, and $\sigma_{1} \geq \sigma_{2} \geq \cdots \geq \sigma_{r}>0$,

$$
\begin{gathered}
\boldsymbol{A}=\boldsymbol{U} \sum \boldsymbol{V}^{T} \\
\sum=\operatorname{diag}\left(\sigma_{1}, \sigma_{2}, \cdots, \sigma_{r}, 0, \cdots, 0\right),
\end{gathered}
$$

(*) is called singular value decomposition of matrix $\boldsymbol{A}$.

SVD has the following important properties. In [14] these important properties were proved. Now we list these properties as follows: stability, transposition invariance, rotation invariance, proportion invariance, mirror transform invariance.

Using singular value decomposition method to do pattern recognition is a commonly used face recognition methods. Du Gan [15] thought that the traditional approach based on SVD only used global information of 
the image, if we divided the face into different regions, the local information of the face can be used to better describe the facial features and improve the recognition rate.

\section{Research about Light Information of Picture}

\subsection{Suppose}

Fast Fourier Transform (FFT) of the image can convert image information into the frequency domain space, we can obtain its amplitude spectrum and phase spectrum. After a Fourier decomposition of the image, the phase spectrum contains texture and structure information about the image, the amplitude spectrum saves contrast illumination information of the image [9].

When we do singular value decomposition of the image, firstly transform image into digital information, it is a gray value matrix in the computer, then the digital matrix is done singular value decomposition, we can get three digital matrixes $\boldsymbol{U}, \boldsymbol{S}, \boldsymbol{V}$. We will set up a group of experiments to verify relation between $\boldsymbol{S}$ matrix and Amplitude spectrum and phase spectrum matrix.

We take the following 20 images that are shown in Figure 1 of ORL [16]. Face database as an example, respectively do FFT and SVD, we can obtain their phase spectrum matrix, amplitude spectrum matrix and $S$ matrix. We have known that the phase spectrum and the amplitude spectrum contain information of image, which information could the $\boldsymbol{S}$ matrix carry?

\subsection{Experiment}

Experiment 1:

Extract amplitude spectrum matrix $\boldsymbol{A}_{\mathrm{i}}$ of each image, find the cosine $K_{i}$ between the amplitude spectrum matrix and $\boldsymbol{S}_{i}$ matrix, and then obtain the angle between them.

We have obtained $K_{i} \quad(i=1,2, \cdots, 20)$ by using matlab tool, Table 1 is the solution.

Experiment 2:

Suppose the cosine of each image between the phase spectrum matrix and $\boldsymbol{S}$ matrix $\boldsymbol{S}$ is $s_{i}(i=1,2, \cdots, 20)$, we have obtained by using matlab tool, Table 2 is the solution.

Experiment 3:

Reconstruct the phase spectrum and amplitude spectrum of the Fourier transform image, and then do inverse Fourier transform, at last compare $\boldsymbol{S}$ matrix with it. We find the cosine between them, the result is $T_{i}(i=1,2, \cdots, 20)$ that are shown in Table 3.

\subsection{Test Results Analysis}

Experiment 1:

We can see that the cosine $K_{i}$ is very close to 1 , the angle

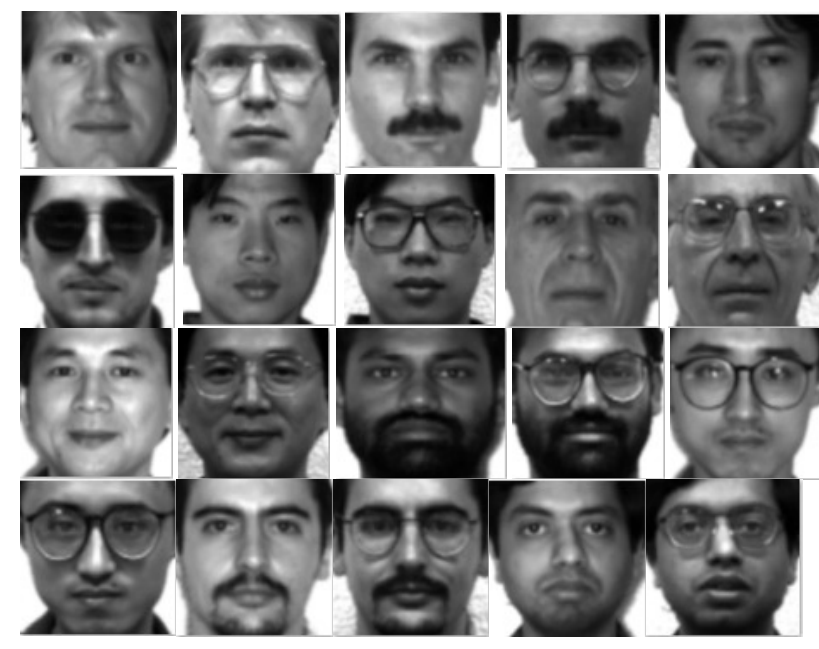

Figure 1. 20 images from ORL face database.

Table $1 . K_{i}$ between amplitude spectrum and $S_{i}$ matrix.

\begin{tabular}{ccccc}
\hline$K_{1}$ & $K_{2}$ & $K_{3}$ & $K_{4}$ & $K_{5}$ \\
0.9297 & 0.9236 & 0.9208 & 0.8561 & 0.8536 \\
$K_{6}$ & $K_{7}$ & $K_{8}$ & $K_{9}$ & $K_{10}$ \\
0.7924 & 0.8754 & 0.8336 & 0.9552 & 0.9198 \\
$K_{11}$ & $K_{12}$ & $K_{13}$ & $K_{14}$ & $K_{15}$ \\
0.9380 & 0.8715 & 0.8572 & 0.7759 & 0.9271 \\
$K_{16}$ & $K_{17}$ & $K_{18}$ & $K_{19}$ & $K_{20}$ \\
0.8715 & 0.9135 & 0.8519 & 0.8502 & 0.8049 \\
\hline
\end{tabular}

Table 2. $K_{i}$ between phase spectrum and $S_{i}$ matrix.

\begin{tabular}{ccccc}
\hline$s_{1}$ & $s_{2}$ & $s_{3}$ & $s_{4}$ & $s_{5}$ \\
0 & 0 & 0 & 0 & 0 \\
$s_{6}$ & $s_{7}$ & $s_{8}$ & $s_{9}$ & $s_{10}$ \\
0 & 0 & 0 & 0 & 0 \\
$s_{11}$ & $s_{12}$ & $s_{13}$ & $s_{14}$ & $s_{15}$ \\
0 & 0 & 0 & 0 & 0 \\
$s_{16}$ & $s_{17}$ & $s_{18}$ & $s_{19}$ & $s_{20}$ \\
0 & 0 & 0 & 0 & 0 \\
\hline
\end{tabular}

Table $3 . K_{i}$ between reconstruct matrix and $S_{i}$ matrix.

\begin{tabular}{ccccc}
\hline$T_{1}$ & $T_{2}$ & $T_{3}$ & $T_{4}$ & $T_{5}$ \\
0.1456 & 0.1632 & 0.1273 & 0.1440 & 0.1337 \\
$T_{6}$ & $T_{7}$ & $T_{8}$ & $T_{9}$ & $T_{10}$ \\
0.1284 & 0.1512 & 0.1352 & 0.1732 & 0.1943 \\
$T_{11}$ & $T_{12}$ & $T_{13}$ & $T_{14}$ & $T_{15}$ \\
0.1284 & 0.1695 & 0.1739 & 0.1773 & 0.1607 \\
$T_{16}$ & $T_{17}$ & $T_{18}$ & $T_{19}$ & $T_{20}$ \\
0.1617 & 0.1507 & 0.1384 & 0.1547 & 0.1579 \\
\hline
\end{tabular}


between the two matrices are close to 0 , the information carried by two matrix is very close, and the amplitude spectrum of image contain contrast illumination information of image.

Experiment 2:

The cosine $s_{i}$ is 0 in the Table 2, the information that two matrixes carried is completely different. The phase spectrum of the image contains texture information of images.

Experiment 3:

After the amplitude spectrum and phase spectrum are reorganized, the matrix contain texture information and contrast illumination information, when we compare it with $\boldsymbol{S}$, we get the cosine of the angle is very small in the Table 3. This shows that the singular value matrix $\boldsymbol{S}$ carries a kind of information.

Through the above experiment, we can conclude that $\boldsymbol{S}$ matrix does not contain texture information; it carries contrast illumination information of image.

\section{Conclusion}

Because of the change of gesture, light, age and other factors face recognition technology in the adaptability has been facing with severe challenge [17]. Present research focus is how to avoid or minimize the effects of light, how to improve the recognition rate at complex background and poor conditions. The key to face recognition is to explore new algorithms and approaches. Face recognition is a challenging task, using an existing method is difficult to obtain good recognition results, how to combine with other technologies, how to improve the recognition rate and recognition speed, reduce computation and improve the robustness, how to use the embedded hardware, all are the future research directions. In the future, our research focus is to do some innovative of approaches and algorithms.

\section{REFERENCES}

[1] F. Tang, J. Wang and Y. W. Guo, "Facial Feature Detection with Hierarchical Constraints," Zhejiang University, Hangzhou, 2004.

[2] S. Lawrence, C. L. Giles and A. C. Tsoi, "Face Recognition: A Convolutional Neural Network Approach," IEEE Transactions on Neural Networks, Vol. 8, No. 1, 1997, pp. 98-113. doi:10.1109/72.554195

[3] H. Peng and X. Y. Zhang, "Face Recognition Using DCTBased Feature Vector," IEEE International Conference, Vol. 4, 1996, pp. 2144-2147.
[4] D. L. Swets and J. J. Weng, "Using Discriminant Eigenfeatures for Image Retrieval," IEEE Transactions on Pattern Analysis and Machine Intelligence, Vol. 18, No. 8, 1996, pp. 831-836.

[5] P. N. Belhumeur, J. P. Hespanha and D. J. Kriegman, "Eigenfaces vs Fisherfaces: Recognition Using Class Specific Linear Projection," IEEE Transaction on PAML, Vol. 19, No. 7, 1997, pp. 711-720. doi:10.1109/34.598228

[6] T. F. Cootes, G. J. Edwards and C. J. Taylor, "Active Appearance Models," Proceeding of 5th European Conference on Computer Vision, 1998, pp. 484-498.

[7] X. W. Hou, S. Z. Li, H. J. Zhang and Q. S. Cheng, "Direct Appearance Models," Proceedings of IEEE International Conference on Computer Vision and Pattern Recognition, Hawaii, December 2001.

[8] W. Zhao, et al., "Face Recognition: A Literature Survey," ACM Computing Surveys, Vol. 35. No. 4, 2003, pp. 399-458. doi:10.1145/954339.954342

[9] Y. X. Lv, Z. Q. Liu and X. H. Zhu, "Image Feature Detection Based on the Principle of Phase Coherency," Ninth National Youth Communication Conference Proceedings, Electronics Industry Press, Beijing, 2004, pp. 1101-1105.

[10] J. Zhang, Y. Yan and M. Lades, "Face Recognition: Eigenface, Elastic Matching, and Neural Nets," Proceedings of the IEEE, Vol. 85, No. 9, 1997, pp. 312-325.

[11] Z. Hang, "Algebraic Feature Extraction for Recognition," Pattern Recognition, Vol. 24, No. 3, 1991, pp. 211-219. doi:10.1016/0031-3203(91)90063-B

[12] S. Romdhani, "Face Recognition Using Principle Components Analysis," MS Dissertation, University of Glasgow, Glasgow, 1997.

[13] R. Brunelli and D. Falavigna, "Person Identification Using Multiple Cues," IEEE Transactions on Pattern Analysis and Machine Intelligence, Vol. 17, No. 10, 1995, pp. 968-985. doi:10.1109/34.464560

[14] M. S. Bartlell, J. R. Movellan and T. J. Se Jnow Ski, "Face Recognition by Independent Component Analysis," IEEE Transon Neural Networks, Vol. 13, No. 6, 2002, pp. 14501464. doi:10.1109/TNN.2002.804287

[15] G. Du and W. J. Zhu, "Face Recognition Method Based on Singular Value Decomposition and Fuzzy Decision," Journal of Image and Graphics, Vol. 11, No. 10, 2006, pp. 1456-1459.

[16] ORL Database. http://d.download.csdn.net/down/2451660/cx_lee

[17] G. D. Su, "Questions about the Application of Face Recognition Technology," China Security, No. 7, 2008, pp. 81-83. 